\title{
BIOLOGÍA REPRODUCTIVA DEL MOLUSCO UNIVALVO Thais chocolata (DUCLÓS, 1832)
}

\author{
Roberto Castellanos Cabrera ${ }^{1}$ \\ Manuela Cosavalente Sánchez ${ }^{2}$
}

\author{
$R E S U M E N$
}

\begin{abstract}
El estudio reproductivo de Thais chocolata (Duclós,1832), fue realizado entre marzo 1992 y febrero 1993. El material biológico estuvo constituido por 1800 ejemplares obtenidos mensualmente de los bancos naturales ubicados entre Puerto de Ilo y Punta Coles (Moquegua). El ciclo reproductivo comprende tres estadios: previtelogénesis (abril, junio y octubre); vitelogénesis (marzo-abril, junio a febrero), y el estadío de postura (en mayo en mayor porcentaje, y en agosto y noviembre en menor porcentaje). La proporción sexual fue de 1:1 y la talla máxima establecida de $88.5 \mathrm{~mm}$. La primera madurez sexual se presentó a los $40 \mathrm{~mm}$ de longitud total, donde la longitud promedio anual del estado maduro es de $57.5 \mathrm{~mm}$.
\end{abstract}

\section{A B S TRACT}

The reproductive study of Thais chocolata (Duclós,1832) was produced between march 1992 and febraury 1993. The biological material constituted 1800 examples obtained monthly from the natural banks located between the Port of Ilo and Punta Coles (Moquegua). The reproductive cycle has three states: previtelogenesis (april, june and october), vitelogenesis (march-april, june to february) and the state of posture (the majority in may, and a laver percentage in august and november). The sexual proportion was $1: 1$ and the maximun length found was $88.5 \mathrm{~mm}$. The first sexual maturity appeared at $40 \mathrm{~mm}$ of total length, where the average annual length in a mature state was $57.5 \mathrm{~mm}$.

\section{INTRODUCCIÓN}

El caracol Thais chocolata (Duclós, 1832) constituye un recurso marino renovable cuya importancia económica a determinado que sea intensamente explotado en los últimos años; situación que ha permitido realizar el estudio reproductivo de esta especie, a fin de regularizar su extracción en el litoral de los departamentos de Tacna y Moquegua.

El estudio reproductivo se ha dirigido esencialmente a determinar el tamaño de la primera madurez sexual y el periodo de postura, con el objeto de establecer el tamaño mínimo de extracción y la época de veda, respectivamente. Aunque estos trabajos se realizaron en 1992 y parte del 1993, no ha tenido una repercusión en el ambiente consumidor de esta especie. El presente estudio ha seguido el mismo procedimiento empleado para Concholepas concholepas (Bruguiére, 1789), puesto que son especies de la familia Muricidae y presentan características similares en su estructura gonadal.

El recurso caracol Thais chocolata (Duclós, 1832) es un prosobranquio univalvo muy abundante en el litoral de los departamentos de Tacna y Moquegua. Su distribución comprende desde Punta Icuy hasta Punta Yerba Buena. Generalmente, se le encuentra 
habitando los fondos cascajo arenoso, conjuntamente con el "choro" Aulacomya ater del cual se alimenta. Los principales lugares de extracción se localizan en Punta Coles, Punta Gentilar, Punta Cata Catas, Puerto Inglés, Tres Hermanas, Pocoma y la Ensillada.

El desembarque se realiza en el Puerto de Ilo, donde es adquirido por las empresas dedicadas a la fase de congelado; es desconchado y la parte comestible se congela y se transporta a Lima para su exportación. Al igual que el recurso "chanque", la vulnerabilidad del stock es total a los aparejos y métodos de extracción. Para la extracción de esta especie se selecciona primeramente en el área de pesca, condicionado por la predominancia de ejemplares de talla comercial. La modalidad de su explotación está en relación a las necesidades de la industria de congelado, lo que determina que sea susceptible a una sobre explotación.

\section{MATERIALES Y MÉTODOS}

El material biológico lo constituyeron 1,800 ejemplares de Thais chocolata (Duclós, 1832) extraídos mensualmente, desde marzo de 1991 hasta febrero de 1992. Los muestreos poblacionales se han realizado en diferentes lugares de la zona; empleando para su extracción el método de buceo libre o Scuba, auxiliado de una embarcación con motor fuera de borda, debido a que la ubicación del banco se encontró a 800 m de la costa.

Las muestras para los estudios reproductivos fueron obtenidas por muestreo estratificado aleatorio de la muestra poblacional, considerando estratos de longitudes totales de $5 \mathrm{~mm}$ de intervalo y un número promedio representativo de 25 ejemplares por cada mes. En total se han colectado 285 ejemplares hembras de Thais chocolata para los estudios reproductivos, obteniendo los parámetros biométricos referentes a longitud total $(\mathrm{mm})$, peso total $(\mathrm{g})$ y peso comestible $(\mathrm{g})$.

El sexo fue determinado por observación directa; y el estado sexual de cada ejemplar se ha obtenido por tratamiento histológico gonadal, siguiendo la metodología establecida por Difiore (1969), con ligeras modificaciones. El análisis histológico de las muestras gonadales tratadas se ha realizado utilizando la escala de madurez sexual de Ramorino (1975).

\section{RESULTADOS}

\section{PRIMERA MADUREZ SEXUAL}

Los resultados obtenidos a través del tratamiento histológico y gonadal de ejemplares hembras de Thais chocolata indican que las mayores frecuencias del estado de máxima madurez sexual se encuentran entre los meses de marzo, julio, enero y febrero; y las menores frecuencias observadas, en mayo, presentando un porcentaje máximo de madurez sexual en la longitud de $57.5 \mathrm{~mm}$. Se observa además que el estado de madurez máxima se presenta a partir de la clase comprendida entre las longitudes totales de 40.0 $44.9 \mathrm{~mm}$. La clase que representa un mayor número de ejemplares, en estado de máxima madurez, se encuentra entre las longitudes de $55.0-59.9 \mathrm{~mm}$.

\section{PERIODO DE DESOVE O PUESTA}

La tabla 1 muestra los estados de desarrollo gonadal para esta especie, observándose que la mayor frecuencia del término de postura se presenta en los meses de mayo, agosto y noviembre-diciembre.

\section{ESTADOS DE DESARROLLO GONADAL}

Según la escala de madurez sexual utilizada para establecer los estados de desarrollo gonadal de Thais chocolata, muestran tres estadios sexuales (Fig.1), siendo estos los siguientes:

\section{Previtelogénesis}

Se presenta con mayor incidencia durante los meses de abril, junio y octubre (Tabla 1, Fig.2), representando el $4.9 \%$ de la muestra total. La Microfotografía A muestra un corte longitudinal del ovario con ovocitos adheridos al epitelio germinativo por medio de un pedúnculo, confiriendo un aspecto piriforme, observándose además las primeras plaquetas vitelinas. El núcleo de cada ovocito ocupa una posición central con un nucleolo bastante nítido.

\section{Vitelogénesis}

Este estado se manifiesta notoriamente durante todos los meses del año, con la excepción del mes de mayo, (Tabla 1, Fig.3) representando el $74.7 \%$ del total de la muestra. La Microfotografía B muestra algunos ovocitos desprendiéndose de las trabéculas, 
aunque la mayoría se encuentra en proceso de desarrollo con tendencia a la forma ovoide, presentando aún los pedúnculos. El núcleo es central conteniendo un nucleolo bastante definido. Las plaquetas vitelinas también están presentes en el citoplasma.

\section{Término de postura}

Constituye el último estado sexual con una representación del $20.4 \%$ de la muestra total y los meses de mayor frecuencia son mayo y noviembre; inclusive en el mes de agosto (Tabla 1, Fig.4). La Microfotografía $\mathrm{C}$ muestra óvulos citolizados y restos de vitelo; además de ovocitos adheridos a las paredes foliculares. El estado de postura en el caracol se presenta durante todo el año con mayor frecuencia en mayo y novie nbre.

Tabla 1. Estado de Desarrollo Gonadal en Hembras de Thais chocolata. Fecha de muestreo: marzo 1992 - febrero 1993.

\begin{tabular}{|c|c|c|c|c|c|c|c|}
\hline \multirow{3}{*}{ MESES } & \multicolumn{7}{|c|}{ ESTADIOS } \\
\hline & \multirow{2}{*}{$\mathrm{N}$} & \multicolumn{2}{|c|}{$\begin{array}{l}\text { PREVITELO- } \\
\text { GENESIS }\end{array}$} & \multicolumn{2}{|c|}{$\begin{array}{l}\text { VITELO- } \\
\text { GENESIS }\end{array}$} & \multicolumn{2}{|c|}{ POSTURA } \\
\hline & & $\mathrm{n}$ & $\%$ & $\mathrm{n}$ & $\%$ & $\mathrm{n}$ & $\%$ \\
\hline Marzo & 26 & 0 & 0.0 & 25 & 96.2 & 1 & 3.8 \\
\hline Abril & 24 & 3 & 12.5 & 20 & 83.3 & 1 & 4.2 \\
\hline Mayo & 21 & 0 & 0.0 & 1 & 4.8 & 20 & 95.2 \\
\hline Junio & 17 & 3 & 17.6 & 10 & 58.8 & 4 & 23.5 \\
\hline Julio & 26 & 2 & 7.7 & 24 & 92.9 & 0 & 0.0 \\
\hline Agosto & 28 & 0 & 0.0 & 20 & 71.4 & 8 & 28.6 \\
\hline Setiembre & 26 & 1 & 3.8 & 19 & 73.1 & 6 & 23.1 \\
\hline Octubre & 32 & 5 & 15.6 & 25 & 78.1 & 2 & 6.3 \\
\hline Noviembre & 25 & 0 & 0.0 & 16 & 64.0 & 9 & 36.0 \\
\hline Diciembre & 21 & 0 & 0.0 & 15 & 71.4 & 6 & 28.6 \\
\hline Enero & 19 & 0 & 0.0 & 18 & 94.7 & 1 & 5.3 \\
\hline Febrero & 20 & 0 & 0.0 & 20 & 100.0 & 0 & 0.0 \\
\hline TOTALES & 28 & 14 & & 213 & & 58 & \\
\hline$\%$ & 10 & 4.9 & & 74.7 & & 20.4 & \\
\hline
\end{tabular}

\section{ESTRUCTURA POBLACIONAL}

Lapoblación del caracol Thais chocolata (Duclós, 1832), durante el período de muestreo (marzo 1992, febrero 1993) presentó una estructura, en base a su tamaño, casi unimodal; es decir, constituida por una población casi uniforme compuesta por individuos con tallas entre 35.0 y $85.0 \mathrm{~mm}$. de longitud total.

La Tabla 2 muestra los rangos de tamaños encontrados en el caracol durante el período de muestreo (marzo 1992 - febrero 1993), indicándose como rango menor el comprendido entre las longitudes 27.0 y 29.9 $\mathrm{mm}$., y como rango mayor $78.0-80.9 \mathrm{~mm}$.; esto nos ha permitido obtener las frecuencias que se presentan en cada rango, cuya sumatoria fue de 1800 individuos determinando una estructura anual unimodal. Los tamaños mayormente representados estuvieron presentes entre los rangos 45.0 y $72.0 \mathrm{~mm}$.

Tabla 2. Frecuencias de los Estados Sexuales en Thais chocolata por clases de longitudes totales-Moquegua.

\begin{tabular}{|c|c|c|c|c|c|c|}
\hline \multirow{2}{*}{$\begin{array}{c}\text { LONGITUD } \\
\text { TOTAL } \\
(\mathrm{mm})\end{array}$} & \multicolumn{3}{|c|}{$\begin{array}{c}\text { ER S T A D I O S } \\
\text { GENESIS }\end{array}$} & $\begin{array}{c}\text { S E X U A L E S } \\
\text { GENESIS }\end{array}$ & \multicolumn{2}{c|}{ POSTURA } \\
\cline { 2 - 7 } & $\mathrm{n}$ & $\%$ & $\mathrm{n}$ & $\%$ & $\mathrm{n}$ & $\%$ \\
\hline $27.0-29.9$ & 0 & 0.0 & 1 & 0.5 & 0 & 0.0 \\
\hline $30.0-32.9$ & 0 & 0.0 & 1 & 0.5 & 0 & 0.0 \\
\hline $33.0-35.9$ & 0 & 0.0 & 0 & 0.0 & 0 & 0.0 \\
\hline $36.0-38.9$ & 0 & 0.0 & 0 & 0.0 & 0 & 0.0 \\
\hline $39.0-41.9$ & 0 & 0.0 & 0 & 0.0 & 1 & 1.7 \\
\hline $42.0-44.9$ & 1 & 7.1 & 2 & 0.9 & 3 & 5.2 \\
\hline $45.0-47.9$ & 1 & 7.1 & 11 & 5.2 & 2 & 3.4 \\
\hline $48.0-50.9$ & 1 & 7.1 & 11 & 5.2 & 7 & 12.1 \\
\hline $51.0-53.9$ & 2 & 14.3 & 16 & 7.5 & 6 & 10.3 \\
\hline $54.0-56.9$ & 5 & 35.7 & 28 & 13.1 & 7 & 12.1 \\
\hline $57.0-59.9$ & 2 & 14.3 & 27 & 12.7 & 8 & 13.8 \\
\hline $60.0-62.9$ & 0 & 0.0 & 32 & 15.0 & 8 & 13.8 \\
\hline $63.0-65.9$ & 1 & 7.1 & 21 & 9.8 & 6 & 10.3 \\
\hline $66.0-68.9$ & 0 & 0.0 & 24 & 11.3 & 7 & 12.1 \\
\hline $69.0-71.9$ & 0 & 0.0 & 12 & 5.6 & 1 & 1.7 \\
\hline $72.0-74.9$ & 1 & 7.1 & 14 & 6.6 & 1 & 1.7 \\
\hline $75.0-79.9$ & 0 & 0.0 & 9 & 4.2 & 1 & 1.7 \\
\hline $78.0-80.9$ & 0 & 0.0 & 4 & 1.9 & 0 & 0.0 \\
\hline TOTALES & 14 & 100.0 & 213 & 100.0 & 58 & 100.0 \\
\hline$\%$ & 4.9 & & 74.7 & & 20.4 & \\
\hline
\end{tabular}




\section{PARÁMETROS MORFOMÉTRICOS}

Los parámetros morfométricos considerados en el presente estudio lo constituyen la longitud total promedio y la longitud modal. La primera es consecuencia de la sumatoria de todas las longitudes presentes cada mes, dividida por el número total de individuos y la segunda viene a ser aquella longitud obtenida mediante la aplicación de una fórmula adecuada.

La longitud total promedio de cada muestra ha sido obtenida mensualmente, presentando cierta variabilidad en relación a la longitud total promedio anual, que fué de $58.5 \mathrm{~mm}$ y casi coincidente con el tamaño mínimo de extracción indicado anteriormente; pero es necesario sostener que esta similitud podría deberse a la presencia de individuos menores. Las longitudes totales promedios durante el año variaron entre 48.7 y $65.9 \mathrm{~mm}$.

\section{PARÁMETROS GRAVIMÉTRICOS}

Entre los principales parámetros gravimétricos determinados en el "caracol" Thais chocolata, se consideran los pesos totales y pesos comestibles expresados en gramos. La variación cuantitativa mensual de dichos parámetros se pone de manifiesto en que el peso total promedio para todo el año es de $47.3 \mathrm{~g}$., y la variación existente de los pesos se halla comprendido entre 28.0 y $70.1 \mathrm{~g}$. El peso comestible. promedio para todo el año es de $12.5 \mathrm{~g}$. y la variación existente de los pesos se encuentra entre los 6.4 y $17.3 \mathrm{~g}$.

\section{RELACIÓN PESO - LONGITUD}

La relación existente entre los parámetros morfométricos y gravimétricos en la especie Thais chocolata es muy estrecha, y en el presente trabajo han sido calculadas mediante la ecuación curvilínea potencial de la forma $Y=a X^{b}$, donde $Y$ representa $a$ los pesos y $X$ a las longitudes. Mediante el sistema de programación han sido calculados los valores de los pesos totales, calculados a partir de los datos establecidos. Estos datos estimados corresponden a todo el año y se evidencia gráficamente mediante la ecuación:

$$
\mathrm{Pt}=3.01412 \times 10^{-4} \mathrm{Lt}^{2.91267}
$$

En cuanto al peso comestible, los cálculos teóricos obtenidos a través de los datos se expresan gráficamente mediante la ecuación:

$$
\mathrm{PC}=0.16751 \times 10^{-4} \mathrm{Lt}^{3.27738}
$$

\section{PROPORCIÓN SEXUAL}

La proporción sexual del caracol Thais chocolata DUCLOS 1832, se ha estimado de una muestra total de 1800 individuos, provenientes de diferentes bancos naturales de la zona de llo - Moquegua. Del total de esta muestra, 884 han sido machos (49.1\%) y 916 hembras $(50.9 \%)$. La prueba de Ji-cuadrado $\left(x^{2}\right)$ demuestra que de marzo de 1982 a febrero de 1983 , para el total de la muestra se obtuvo un valor de $\mathrm{x}^{2}=$ 2.085 , que no es significativo a un nivel del $95 \%$, aceptándose la hipótesis de que la proporción sexual de Thais chocolata es de 1:1, siendo el número de grados de libertad $k=1$.

En general, la producción sexual de Thais chocolata resulta de la división entre el número de individuos machos y el número de individuos hembras, dando como resultado el valor de 0.94 , la proporción de machos; resultado de la división del número de individuos machos entre la suma del número de machos y hembras, fue de 0.48 .

\section{DISCUSIÓN}

\section{PRIMERA MADUREZ SEXUAL}

Los resultados obtenidos en Thais chocolata indican que esta especie lograría su primera madurez sexual a partir de $42.5 \mathrm{~mm}$ de longitud total promedio, correspondiendo a dos muestras obtenidas en los meses de marzo y abril. Podríamos aseverar que esta especie durante los demás meses se encuentra en estado maduro, indicando que solamente en el mes de mayo se presentó porcentaje muy bajo, y en la Fig. 4 puede observarse que existe un elevado porcentaje de término de postura en el mes de mayo. También se indica que el mayor porcentaje de hembras maduras se encuentran entre las longitudes de $57,5 \mathrm{a}$ $62,5 \mathrm{~mm}$., y de acuerdo a esto podría considerarse como límite de tamaño mínimo de extracción a partir de los $60,0 \mathrm{~mm}$ de longitud total.

\section{PERIODO DE DESOVE O PUESTA}

Durante el presente estudio se ha determinado que la mayor frecuencia del término de postura se observa en el mes de mayo; otro pico de menor 
frecuencia se observa en el mes de agosto; en consecuencia la determinación de la época de veda para Thais chocolata podría abarcar la época de mayor postura que ocurre entre los meses de abril, mayo y junio, aunque según referencias de mariscadores, esta especie comienza a desovar a partir de los meses de mayo, hasta agosto, y el "arrocillo" en forma separada está presenteenla costa, en los meses de setiembre, octubre y noviembre.

\section{ESTADOS DE DESARROLLO GONADAL}

Durante el período de muestreose indicaque Thais chocolata, presenta un ciclo estacional que comprende todo el año, con estado de desarrollo ovárico contínuo, no existiendo un estado de verdadero reposo; aunque en la Tabla 1 , se muestra que los estados de previtelogénesis y de término de postura, se manifiesta con mayor frecuencia en los meses posteriores a marzo, hasta julio. El estado de vitelogénesis se encuentra presente en todo el año con la excepción antes indicada.

\section{REFERENCIAS BIBLIOGRÁFICAS}

AVILES, S. y E.LOZADA, 1975. Estudio histológico del ciclo reproductivo de Concholepas concholepas (Bruguiere, 1789) en Punta Saliente, Coquimbo. Bol. Soc. Biol. de Concepción, 44: 207-218.

CASTELlANOS, R. \& COSAVALENTE, M. 1995. Biología reproductiva del molusco univalvo Concholepas concholepas Brugiere, 1789. Ciencia \& Desarrollo 2:7-11.UNJBG.

CASTILLA, J.C. 1974. Notes on the mating behavior of Concholepas concholepas (Mollusca: Gastropoda: muricidas) from chile. Veliger, 16(3): 292.

CASTILLA, J.C. y J. CANCINO, 1976. Spawning behaviour and egg capsules of Concholepas concholepas (Mollusca: Gastropoda: Muricidas). Marine Biology, 37: 255 - 263.

CASTILLA, J.C. 1979. Concholepas concholepas (Mollusca: Gastropoda: Muricidae) Postura de cápsula en el laboratorio y en la naturaleza. Biología Pesquera Chile 12 : 91 - 97.

DIFIORE, M.S. 1969. Diagnóstico histológico. Tomo I. Sexta Edición. Editorial El Ateneo. Buenos Aires. Argentina. pp.4897

DIRECCION REGIONAL DE PESQUERIA, 1980. Proyecto: Evaluación de los recursos hidrobiológicos costeros (mariscos) Tacna-Moquegua. Vol. II. Estudio poblacional. Tacna, Perú.

\section{CONCLUSIONES}

- La primera madurez sexual se presentó a partir de los $40 \mathrm{~mm}$. de longitud total, siendo la longitud promedio anual del estado maduro de $57,5 \mathrm{~mm}$.

- El período de desove o postura mayor comprendió el mes de mayo, y períodos significativos en agosto y noviembre.

- El ciclo reproductivo anual abarcó el estado de previtelogénesis (abril, junio y octubre), vitelogénesis (marzo-abril; julio, octubre y febrero) y término de postura (marzo, incluso junio y octubre).

- La proporción sexual fué de 1:1 y la talla máxima encontrada fué de $88.5 \mathrm{~mm}$.

\section{RECOMENDACIONES}

- Establecer el período de veda durante los meses de abril-mayo-junio, aunque para esta especie no es necesario puesto que los extractores seleccionan el tamaño comerciable.

- La talla mínima de extracción debe estar cornprendida entre 55 y $60 \mathrm{~mm}$ de longitud total, medida desde el ápice y el borde anterior de canal sifonal.

DIRECCIÓN ZONAL DE PESQUERIA ILO, 1982. Proyecto: Evaluación de Recursos Hidrobiológicos (Mariscos) Moquegua. Vol.III. Estudio reproductivo. Moquegua - Perú.

GALLARDO, S. C. 1973. Desarrollo intrapsular de Concholepas concholepas (Gastrópoda: Muricidas). Museo Nacional de Historia Natural, Santiago de Chile, $16: 1 \cdot 16$

GUZMAN, C.E.; AMIN, M. y M.DELPIN, 1972. Análisis histológico del sistema reproductor masculino de Concholepas concholepas Bruguiére, 1789. Bol. Soc. Biol. de Concepción. $45: 117-127$.

HUAQUIN,L.1969. Análisis histológico del ovario de Concholepas concholepas (Bruguiére, 1789). (Gastrópoda: Muricidas). Biologia Pesquera Chile 12 : 71 - 77.

RAMORINO, L. 1975. Ciclo reproductivo de Concholepas concholepas en la zona de Valparaíso. Rev.Biol.Mar. Valparaiso, 15 (2):149-177.

RAMORINO, L. 1980. Recurso "Ioco" Concholepas concholepas (Bruguiére) (Gastrópoda: Nonotocardia: Muricidae) en: Estado Actual de las Principales Pesquerias Nacionales. Bases para un desarrollo pesquero. Corporación de Fomento de la Producción. Instituto de Fomento Pesquero de Chile. 
MICROFOTOGRAFÍA A: Previtelogénesis chocolata DUCLOS.

MICROFOTOGRAFIA B: Vitelogénesis en ovario de Thais chocolata DUCLOS

MICROFOTOGRAFIA C: Postura o desove en ovario de Thais chocolata DUCLOS. en ovario de Thais
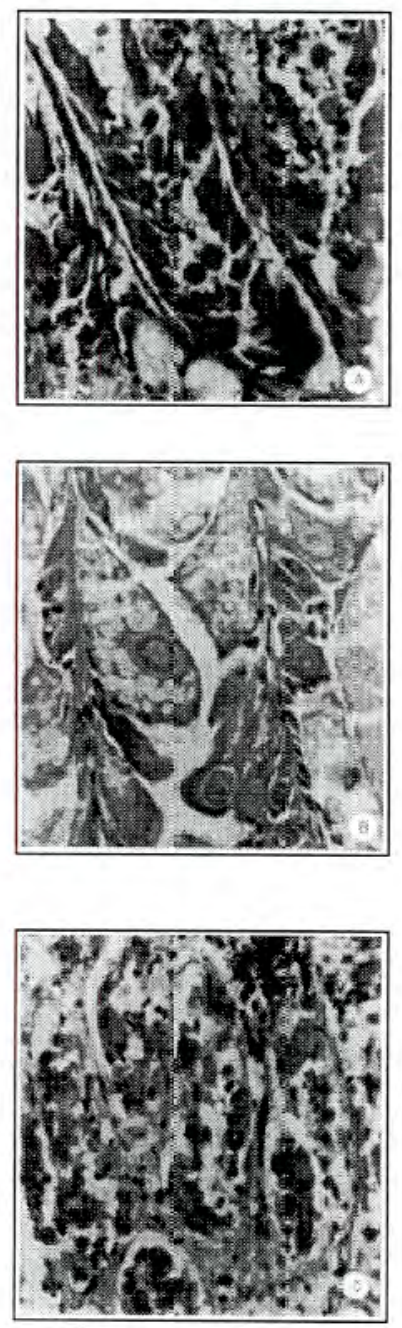

Fig. 1. Ciclo Reproductivo de Thais chocolata. Marzo 1992. Febrero 1993.

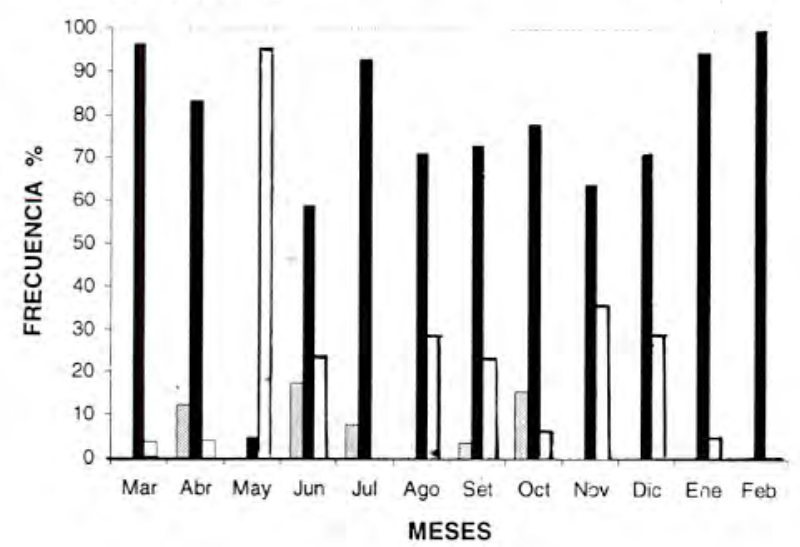

口PREVITEOGENESIS — VITELOGENESIS $\square$ POSTURA
Fig.2. Previtelogenesis en Thais chocolata. Mar-a 1092 Febrero 1993.

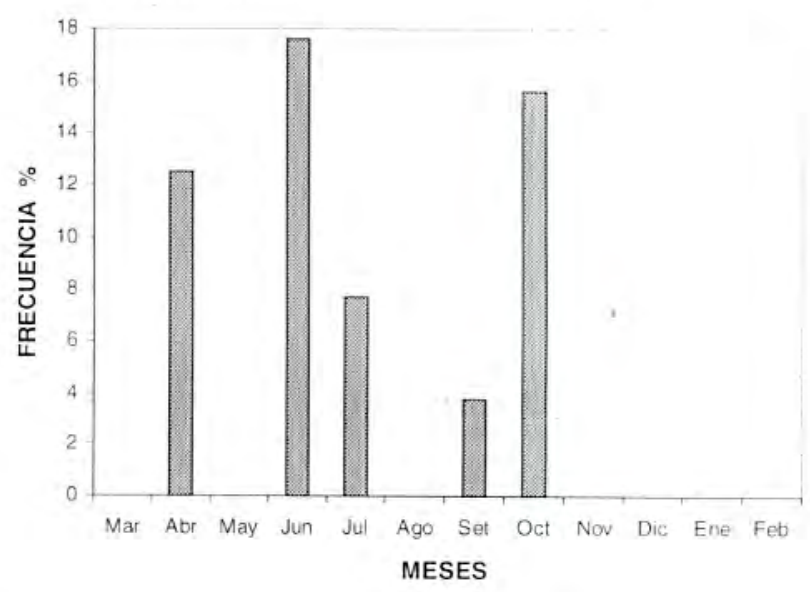

Fig.3. Vitelogenesis en Thais chocolata. Marzo 1992 - Fethero 1993.

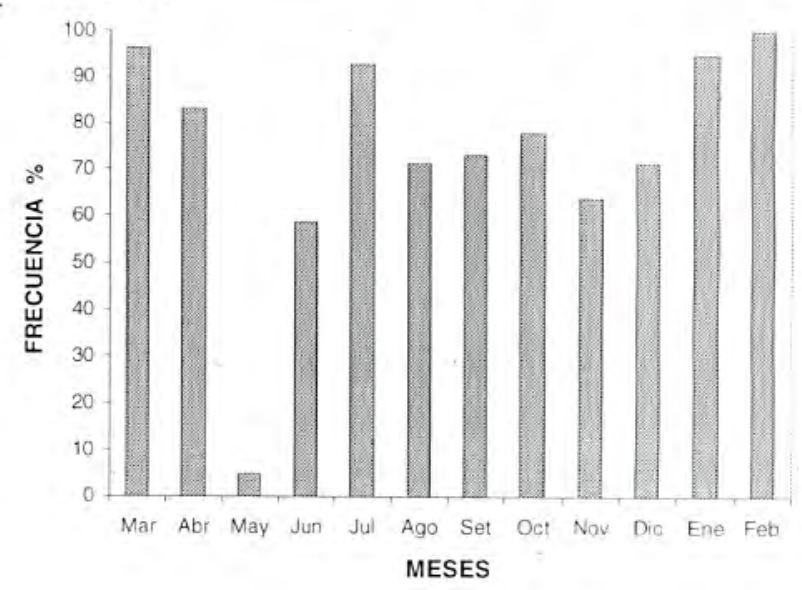

Fig.4. Tiempo de Postura en Thais chocolata. Marzo 1992. Febrero 1993.

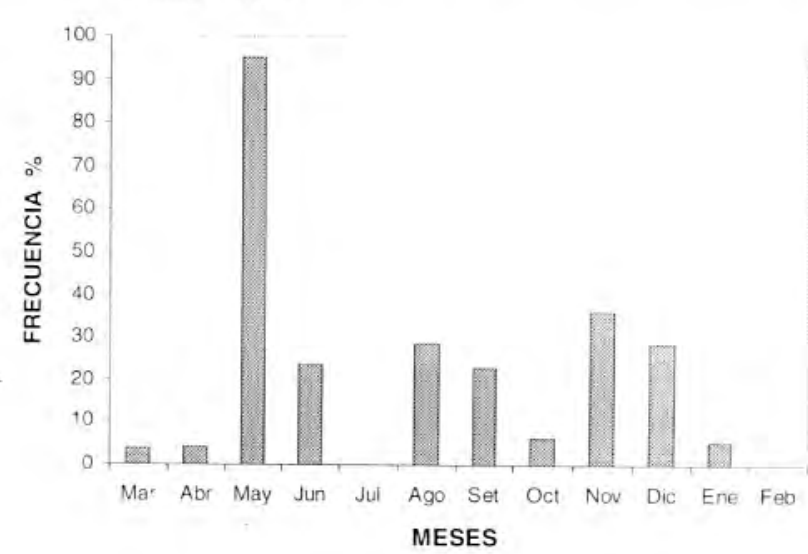

